\title{
A SURVEY OF MEDICAL IMAGE PROCESSING TOOLS
}

\author{
Lee Lay Khoon, Liew Siau Chuin
}

\author{
Faculty of Computer Systems \& Software Engineering \\ Universiti Malaysia Pahang, 26300 Gambang, Kuantan, \\ Pahang Darul Makmur, Malaysia \\ E-mail: jesscefyn@hotmail.com, liewsc@ump.edu.my
}

\begin{abstract}
A precise analysis of medical image is an important stage in the contouring phase throughout radiotherapy preparation. Medical images are mostly used as radiographic techniques in diagnosis, clinical studies and treatment planning. Medical image processing tool are also similarly as important. With a medical image processing tool, it is possible to speed up and enhance the operation of the analysis of the medical image. This paper describes medical image processing software tool which attempts to secure the same kind of programmability advantage for exploring applications of the pipelined processors. Terminology and important issues in image analysis are first presented. These tools simulate complete systems consisting of several of the proposed processing components, in a configuration described by a graphical schematic diagram. In this paper, fifteen different medical image processing tools will be compared in several aspects. The main objective of the comparison is to gather and analysis the tool in order to recommend users of different operating systems on what type of medical image tools to be used when analysing different types of imaging. A result table was attached and discussed in the paper and concluded with a discussion on the future of tool in biomedical or computer vision research.
\end{abstract}

Keywords: Image processing; Medical Image; Processing Tool

\section{INTRODUCTION}

Image Processing is a form of information processing where the input and output are images, such as photographs or frames of video. Image Processing techniques usually process images as 2D signals and apply standard signal processing techniques to them. In general, image processing can be divided into digital image processing and medical image processing. . This paper will focus on medical image processing tools. In medical fields nowadays, medical imaging and processing tools are playing crucial roles in many applications. According to Lee and Liew (2015), applications usually take place throughout the clinical track of events; not only within diagnostic settings, but prominently in the area of preparation, carrying out and evaluation before surgical operations. Therefore, the pros and cons of the medical image will directly influence the result of the diagnosis from a doctor to the patient. Besides that, medical imaging itself have noise and speckle like ultrasound, thus it will increase the difficulties of doctors' judgment. The goal of this paper is to gather up and analysis a variety of medial image processing tools in the market. To figure out which tools is open source, which tools is suitable to run on what operating system. Figure 1 shows a medical image processing tool consists a few functions such as, diffusion, simulation and much more. 


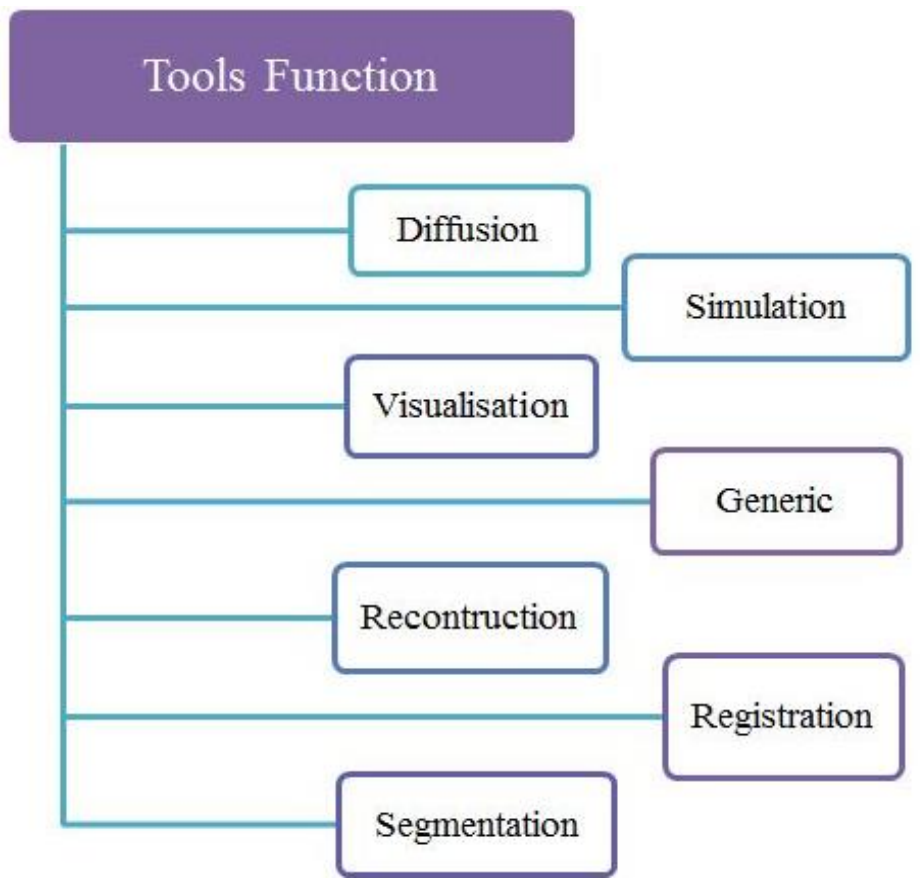

Figure 1. Tools' Function

Many image processing tools have been created to carry out different function like illustrated in Figure 1. Total of seven functions has been included. However there are not all tools can include all the functionality. Thus, this paper focuses on reviewing fifteen types of medical image processing tools and categories in term of functionality or platform.

\section{FOUNDATION}

As a foundation for the following survey and discussion, in this section, a brief introduction on medical image processing tools and medical image processing will be presented. The main functions of a medical image processing tool (MIP) will also be explained in this section.

\section{IMAGE PROCESSING TOOLS}

A major purpose of image processing is to improve the appearance of an image, thus, there is a lot of image processing tools. Image Processing Tools provides assistance to engineers and scientists with an extensive set of plugin, toolkit, functions, and apps for image processing, analysis. Most image-processing techniques involve treating the image as a two-dimensional signal and applying standard signal-processing techniques to it. Figure 2 illustrates the type of image processing. Image processing can be divided into two main types, which is digital image processing tool and medical image processing tools. 


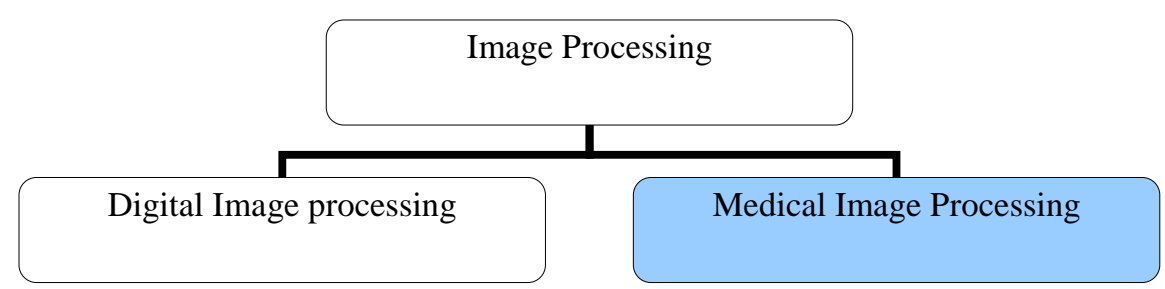

Figure 2. Type of Image Processing

\section{DIGITAL IMAGE PROCESSING TOOLS}

According to Solomon et.al (2011), digital image processing is the process of using computer algorithms to perform image processing on digital images. As a subcategory or field of digital signal processing, digital image processing has many advantages over analog image processing. It allows a much wider range of algorithms to be applied to the input data and can avoid problems such as the build-up of noise and signal distortion during processing. Many of the techniques of digital image processing, or digital picture processing as it often was called, were developed in the at first 1960.

\section{MEDICAL IMAGES}

In the modern medical field, medical imaging has undergone a major advancement. This technology is important as it can be applied before an actual surgery where this has been done by Charge (2013). Over the years, different sorts of medical imaging have been developed, different type of medical image adapts different type of technology. Each of the medical images has their own advantages and disadvantages.

\section{Medical images and their properties}

Medical imaging is the visualization of body parts, tissues or organs for use in clinical diagnosis, treatment and disease monitoring. Imaging techniques encompass the fields of radiology, nuclear medicine and optical imaging. There are few type of medical image such as X-ray. X-ray based methods of medical imaging include conventional X-ray, computed tomography (CT) and mammography. Lu et al (2012) to enhance the X-ray image, contrast agents can be used for example for angiography examinations by. Molecular imaging is used in nuclear medicine and uses a variety of methods to visualize biological processes taking place in the cells of organisms. Small amounts of radioactive markers, called radiopharmaceuticals, are used for molecular imaging. Other types of medical imaging are magnetic resonance imaging (MRI) and ultrasound imaging. Unlike conventional X-ray, CT and Molecular Imaging, MRI and ultrasound operate without ionizing radiation. MRI uses strong magnetic fields, which produce no known irreversible biological effects in humans. For certain types of clinical cases, imaging techniques can be used that do not require the use of ionizing radiation. For example, high-frequency sound waves are used in Ultrasound scans. In magnetic resonance imaging (MRI), radio waves are used together with strong magnetic fields.

\section{Computed Tomography (CT)}

Computed Tomography (CT), also commonly referred to as a CAT scan, is a medical imaging method that combines multiple X-ray projections taken from different angles to produce detailed cross-sectional images of areas inside the body. CT images allow doctors to get very precise, 3$D$ views of certain parts of the body, such as soft tissues, the pelvis, blood vessels, the lungs, the brain, the heart, abdomen and bones. CT is also often the preferred method of diagnosing many cancers, such as liver, lung and pancreatic cancers (Greenberg et al, 2012). 
CT is often used to evaluate the presence, size and location of tumors. In addition, CT also uses to evaluate organs in the pelvis, chest and abdomen. Colon health (CT colongraphy) Vascular condition/blood flow Pulmonary embolism (CT angiography) Abdominal aortic aneurysms (CT angiography). Bone injuries Cardiac tissue Traumatic injuries Cardiovascular disease.

\section{Magnetic Resonance Imaging (MRI)}

Magnetic Resonance Imaging (MRI) is a medical imaging technology that uses radio waves and a magnetic field to create detailed images of organs and tissues. MRI has proven to be highly effective in diagnosing a number of conditions by showing the difference between normal and diseased soft tissues of the body (Stein et al, 1998).

MRI is often used to evaluate: Abnormal tissue in Blood vessels, Breasts Bones and joints Organs in the pelvis, chest and abdomen (heart, liver, kidney, spleen) Spinal injuries Tendon and ligament tears

\section{Positron Emission Tomography (PET)}

Alexander (2014) stated that positron Emission Tomography (PET) is a nuclear imaging technique that provides physicians with information about how tissues and organs are functioning. PET, often used in combination with CT imaging, uses a scanner and a small amount of radiopharmaceuticals which is injected into a patient's vein to assist in making detailed, computerized pictures of areas inside the body.

PET is often used to evaluate:

Neurological diseases such as Alzheimer's and Multiple Sclerosis Cancer Effectiveness of treatments Heart conditions

\section{- PET-CT}

For added precision, physicians use a medical imaging technique that combines PET and CT. This allows images acquired from both devices to be taken sequentially and combined into a single superposed image. PET-CT serves as a prime tool in the delineation of tumor volumes, staging and the preparation of patient treatment plans. The combination has been shown to improve oncologic care by positively impacting active treatment decisions, disease recurrence monitoring and patient outcomes, such as diseasefree progression (Drzezga et al, 2012).

\section{Ultrasound}

Diagnostic ultrasound, also known as medical sonography or ultrasonography, uses high frequency sound waves to create images of the inside of the body. The ultrasound machine sends sound waves into the body and is able to convert the returning sound echoes into a picture. According to Awad et al (2012), Ultrasound technology can also produce audible sounds of blood flow, allowing medical professionals to use both sounds and visuals to assess a patient's health.

Ultrasound is often used to evaluate:

Pregnancy Abnormalities in the heart and blood vessels Organs in the pelvis and abdomen Symptoms of pain, swelling and infection. 


\section{X-ray}

$\mathrm{X}$-ray technology is the oldest and most commonly used form of medical imaging. X-rays use ionizing radiation to produce images of a person's internal structure by sending X-ray beams through the body, which are absorbed in different amounts depending on the density of the material. In addition, included as "x-ray type" devices are also mammography, interventional radiology, computed radiography, digital radiography and computed tomography (CT). In the work of Suryanarayana and Norton (2013), proved that radiation therapy is a type of device which also utilizes either x-rays, gamma rays, electron beams or protons to treat cancer.

$\mathrm{X}$-ray images are typically used to evaluate:

Broken bones Cavities Swallowed objects Lungs Blood vessels Breast (mammography).

\section{Mammography}

Mammography is specialized medical imaging that uses a low-dose $\mathrm{x}$-ray system to see inside the breasts. A mammography exam, called a mammogram, aids in the early detection and diagnosis of breast diseases in women. Digital mammography, also called full-field digital mammography (FFDM), is a mammography system in which the x-ray film is replaced by electronics that convert $\mathrm{x}$-rays into mammographic pictures of the breast. These systems are similar to those found in digital cameras and their efficiency enables better pictures with a lower radiation dose. These images of the breast are transferred to a computer for review by the radiologist and for long term storage. The patient's experience during a digital mammogram is similar to having a conventional film mammogram.

Computer-aided detection (CAD) systems search digitized mammographic images for abnormal areas of density, mass, or calcification that may indicate the presence of cancer by (Berg et al, 2012). The CAD system highlights these areas on the images, alerting the radiologist to carefully assess this area.

\section{MEDICAL IMAGE PROCESSING TOOLS (MIP)}

Medical imaging is the technique, process and art of creating visual representations of the interior of a body for clinical analysis and medical intervention. Medical imaging seeks to reveal internal structures hidden by the skin and bones, as well as to diagnose and treat disease. Medical imaging also establishes a database of normal anatomy and physiology to make it possible to identify abnormalities. Although imaging of removed organs and tissues can be performed for medical reasons, such procedures are usually considered part of pathology instead of medical imaging. Thus, this paper will mainly focus on the survey of medical image processing tools. In the next section a rough overview of different types of image processing tools will be presented. The Figure 3 illustrates the medical fifteen type of image processing tools has been reviewed. 


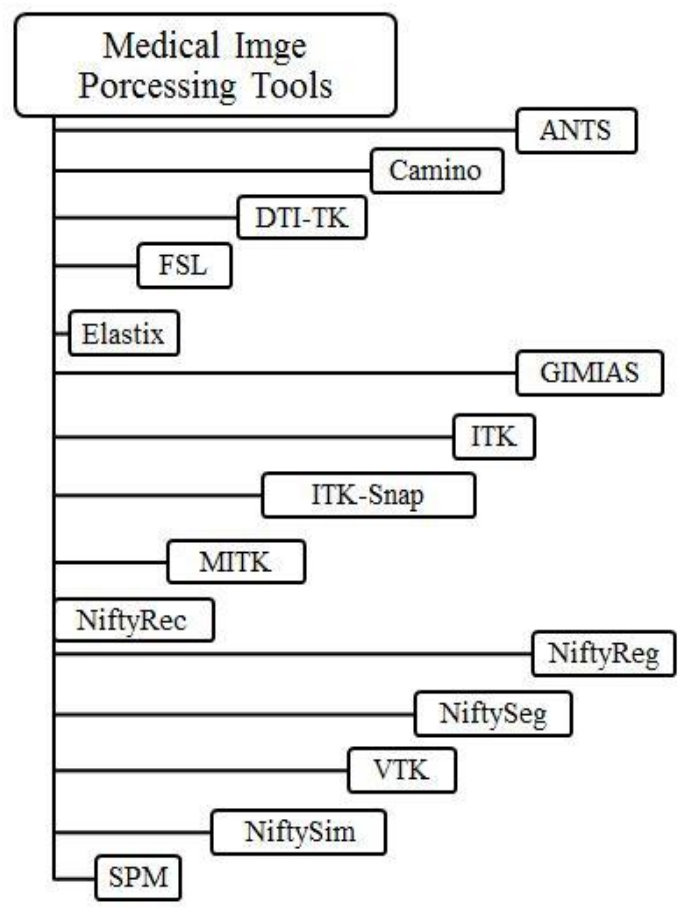

Figure 3. Tools of Medical Image Processing

\section{MULTIPURPOSE SOFTWARE}

Multipurpose machine vision software is considered as software. Sonka et al (2014) found out software, which is heavier than small toolkit programs and may have more functionality integrated in the same package. Multipurpose software can refer as multipurpose toolkit, which carries out of multi-purpose integrated.

\section{SURVEY}

There are several software tools available to perform medical image processing. This following section introduced 15 types of tools that are often used by researchers.

\section{VTK}

The Visualization Toolkit (VTK) is an open-source, freely available software system for 3D computer graphics, image processing and visualization. This tool is by Kitware, whose team currently is still performing future development to the toolkit, this tool offers professional support and consulting services for VTK. Besides that, VTK has an extensive information visualization framework, has a suite of 3D interaction widgets, supports parallel processing, and integrates with various databases on GUI toolkits such as QT and TK (Pettit et al, 2012). Besides that, VTK is permissively licensed, cross-platform toolkit for scientific data processing, visualization, and data analysis. VTK was originally developed for very different graphics card architecture. It is over two decades old, this tool is enhanced Modern graphics cards feature fully programmable, highly parallelized architectures with large core counts. VTK has been implemented in the work of Hanwell et al (2015). VTK's rendering code was rewritten to take advantage of modern graphics cards, maintaining most of the toolkit's programming interfaces. According to Hanwell, VTK offers the opportunity to compare the performance of old and new 
rendering code on the same systems/cards. Significant improvements in rendering speeds and memory footprints mean that scientific data can be visualized in greater detail than ever before. The widespread use of VTK means that these improvements will reap significant benefits.

\section{ITK}

ITK or Insight Segmentation and Registration is an open-source, cross-platform system that provides developers with an extensive suite of software tools for image analysis. ITK has been implemented in the work of Liu et al (2014). In addition, ITK provide leading-edge segmentation and registration algorithm in two, three and more dimensions, it is distributed as an open-source software package by Johnson et al (2014). ITK implements the operation of convolution with the derivatives of a Gaussian filter in a recursive way using approximated 2D Gaussian operators, an approach which is computationally more efficient (TIG, 2014). In addition to the latter, to scan an image region during the region growing phase, ITK defines a so called "image iterator" which is an abstraction of a memory pointer, therefore resulting in more efficient utilization of memory.

\section{FSL}

According to (Smith, 2012), FSL (FMRIB Software Library) is created by Analysis Group, FMRIB, and OXFORD, UK. FSL is a comprehensive library of analysis tools for FMRI, MRI and DTI brain imaging data. FMRIb Software Library (FSL) Brain Extraction Tool (BET) is a widely used algorithm for removing extra cranial tissues from MR images, this tool has been used in the research work of Fagiolo (2014). Fagiolo presented a recursive procedure by using BET that is extremely robust for extracting brain images without any manual intervention. BET is effective and simple to operate. However it often fails to exclude fully all non-brain tissues unless the center of gravity of the brain is specified to the program. In this cases, Fagiolo has been carried out several experiments, tested on 150 adult head volumes and produced accurate results in all cases. Eventually, result indicated that the approach is efficient and suitable for incorporation into automated workflows and other pipeline methods.

\section{SPM}

Statistical Parametric Mapping refers to the construction and assessment of spatially extended statistical processes used to test hypotheses about functional imaging data. These ideas have been instantiated in software that is called SPM. The SPM software package has been designed by Karl Friston for the analysis of brain imaging data sequences, such as fMRI, PET, SPECT, EEG and MEG. SPM has been implemented by Penny et al (2011), in brain abnormalities detection. In the following years, SPM has be used by Sowell et al (2014) to observed statistical parametric maps of gray matter, white matter, and CSF differences between the groups revealed that the subjects with early-onset schizophrenia had larger ventricles, predominantly in the posterior horns of the lateral ventricles, and midcallosal, posterior cingulate, caudate, and thalamic abnormalities. Volumetric analyses of the lateral ventricles in native image data space confirmed significantly higher volume in posterior, but not anterior, regions . Randomization tests confirmed the overall statistical significance of the group differences and validity of the parametric maps.

\section{GIMIAS}

Graphical interface for medical image Analysis and Simulation (GIMIAS) is a workfloworiented environment for solving advanced biomedical image computing and individualized simulation problems, which is extensible through the development of problem-specific plug-ins 
(Larrabide et al, 2009). GIMIAS has been implemented in the research work of (Qasrawi et al, 2015).

According to Qasrawi, electroporation is the phenomenon in which cell membrane permeability is increased by exposing the cell to high intensity electric fields. In living tissues, such permeabilization boost can be used in order to enhance the penetration of drugs or DNA plasmids or to destroy undesirable cells and it is typically performed by applying pulsed high voltages across needle electrodes. When used for ablation, and particularly in the context of liver tumors, it is often claimed that in contrast with thermal ablation techniques, electroporation is not severely impacted by the presence of large blood vessels because the heat sinking characteristic of those is not relevant for the electric field distribution. However, blood vessels do distort the electric field distribution because of their high inner conductivity.

The goal of the study is to numerically analyze the relevance of such distortion in a clinical scenario. An anatomically realistic model of the liver and its vasculature within an abdominal section was implemented. The blood vessels ranged from 0.3 to $6.3 \mathrm{~mm}$ in diameter and from 24 to $68.56 \mathrm{~mm}$ in length. A series of simulations with plausible random locations and depths (needle tips from 10 to $25 \mathrm{~mm}$ within the liver) for the electrode pairs was conducted. Locations and depths were carefully verified so that the electrodes did not penetrate the vessels. The results were compared to those assuming that liver tissue is homogeneous. These comparisons indicate that volume error caused by neglecting the presence of vessels is less than $3 \%$, which can be considered negligible if compared to other error sources. However, it was noticed that if the treated region comprises vessels or is very close to them, undertreated and over treated spots would appear around the vessels. Since undertreated spots may imply that tumor cells remain viable after treatment, caution is advised.

\section{NiftyReg}

NiftyReg is open-source software for efficient medical image registration. It has been mainly developed by member of the Translational Imaging Group with the Centre for Medical Image Computing at University College London, UK (TIG, 2014) . Niftreg has been used in the work of Mengler et al (2014). Rigid and non-rigid registrations were implemented to our in-house software. The non-rigid registration algorithm of the NiftyReg and MIM was based on the freeform deformation. The accuracy of the two software were evaluated when contoured structures to peak-inhale and peak-exhale. 4 Dimension (4D-CT) images data sets were measured using the dice similarity coefficient (DSC). The evaluation was performed in 20 lung SBRT patients. Result indicates NiftReg able to implement in 3D medical image registration.

\section{Elastix}

Elastix is open source software, based on the well-known Insight Segmentation and Registration Toolkit (ITK). The software consists of a collection of algorithms that are commonly used to solve (medical) image registration problems. The modular design of Elastix allows the user to quickly configure, test, and compare different registration methods for a specific application. This study evaluates the performance of the elastix toolbox deformable image registration algorithm for VOI and voxel-wise assessment of longitudinal variations in FDG tumor uptake in NSCLC patients. Evaluation of the elastix toolbox was performed by using 18F-FDG PET/CT at baseline and after 2 cycles of therapy (follow-up) data in advanced NSCLC patients. The elastix toolbox is an integrated part of the IMALYTICS workstation, was used to apply a CT-based non-linear image registration of follow-up PET/CT data using the baseline PET/CT data as reference. Lesion statistics were compared to assess the impact on therapy response assessment. 
Next, CT-based deformable image registration was performed anew on the deformed follow-up PET/CT data using the original follow-up PET/CT data as reference, yielding a realigned follow-up PET dataset. Performance was evaluated by determining the correlation coefficient between original and realigned follow-up PET datasets. The intra- and extra-thoracic tumors were automatically delineated on the original PET using a $41 \%$ of maximum standardized uptake value (SUVmax) adaptive threshold. Equivalence between reference and realigned images was tested (determining 95\% range of the difference) and estimating the percentage of voxel values that fell within that range. A result shows that the thirty-nine patients with 191 tumor lesions were included. In 37/39 and 12/39 patients, respectively, thoracic and non-thoracic lesions were evaluable for response assessment. Using the EORTC/ SUVmax-based criteria, 5/37 patients had a discordant response of thoracic, and 2/12 a discordant response of non-thoracic lesions between the reference and the realigned image. FDG uptake values of corresponding tumor voxels in the original and realigned reference PET correlated well (R2=0.98). Using equivalence testing, $94 \%$ of all the voxel values fell within the $95 \%$ range of the difference between original and realigned reference PET.

From the research work of Kerner et al (2015), the elastix toolbox impacts lesion statistics and therefore therapy response assessment in a clinically significant way. The elastix toolbox is therefore not applicable in its current form and/or standard settings for PET response evaluation. Further optimization and validation of this technique is necessary prior to clinical implementation.

\section{ANTS}

ANTS or Advances Normalization Tools is created by Avants and Song (2009). An ANT is able to extract information from complex Dataset, and it is very useful for managing interpreting and visualizing multidimensional data. The United States National Institutes of Health (NIH) commit significant support to open-source data and software resources in order to foment reproducibility in the biomedical imaging sciences. According to the work of Avants et al (2011), ANTS has been reported and evaluated a recent product of this commitment: Advanced Neuroimaging Tools (ANTs), which is approaching its 2.0 release.

The ANTs open source software library consists of a suite of state-of-the-art image registration, segmentation and template building tools for quantitative morphometric analysis. In this work, they used ANTs to quantify, for the first time, the impact of similarity metrics on the affine and deformable components of a template-based normalization study. They detail the ANTs implementation of three similarity metrics: squared intensity difference, a new and faster cross-correlation, and voxel-wise mutual information. Besides that, they used two-fold crossvalidation to compare their performance on openly available, manually labeled, T1-weighted MRI brain image data of 40 subjects (UCLA's LPBA40 dataset).

Evaluation results have been reported on cortical and whole brain labels for both the affine and deformable components of the registration. Results indicate that the best ANTs methods are competitive with existing brain extraction results (Jaccard $=0.958$ ) and cortical labeling approaches. Mutual information affine mapping combined with cross-correlation diffeomorphic mapping gave the best cortical labeling results (Jaccard $=0.669 \pm 0.022$ ). Furthermore, our two-fold cross-validation allows us to quantify the similarity of templates derived from different subgroups. Our open code, data and evaluation scripts set performance benchmark parameters for this state-of-the-art toolkit. This is the first study to use a consistent transformation framework to provide a reproducible evaluation of the isolated effect of the similarity metric on optimal template construction and brain labeling. 


\section{NiftySeg}

NifySeg is the one of the project developed at University College London, which is licensed under BSD license. It a tool which contains several programs to perform EM based segmentation of image $n$ nifty or analyses format (TIG, 2014). Forney Corporation, MK Engineering (MKE) and NYSEG jointly conducted extensive testing of a new Burner Diagnostic System (BDS) based on analysis of flame turbulence in the burner ignition zone. Tests were conducted on the 700 MW coal-fired units at NYSEG Kintigh Station with the objective to evaluate the new system.

\section{ITK-Snap}

ITK-Snap is a software tool used to segment structures in 3D medical images, it is created by Paul Yushkevich. ITK-SNAP provides semi-automatic segmentation using active contour methods, as well as manual delineation and image navigation (Khesin et al, 1997). Active contour segmentation and its robust implementation using level set methods are well-established theoretical approaches that have been studied thoroughly in the image analysis literature. Despite the existence of these powerful segmentation methods, the needs of clinical research continue to be fulfilled, to a large extent, using slice-by-slice manual tracing. To bridge the gap between methodological advances and clinical routine, Yushkevich et al (2006) developed an open source application called ITK-SNAP, which is intended to make level set segmentation easily accessible to a wide range of users, including those with little or no mathematical expertise. The work of Yushkevich explained the methods and software engineering philosophy behind this new tool and provides the results of validation experiments performed in the context of an ongoing child autism neuroimaging study. The validation establishes SNAP intrarater and interrater reliability and overlap error statistics for the caudate nucleus and finds that SNAP is a highly reliable and efficient alternative to manual tracing. Analogous results for lateral ventricle segmentation are provided.

\section{MITK}

The Medical Imaging Interaction Toolkit or MITK is a software tool that combines the Insight Toolkit (ITK) and the Visualization Toolkit (VTK) with application framework. According to Nolden et al (2013) ITK is under BSD-Style license. MITK allows an easy combination of algorithms developed by ITK with visualizations created by VTK and extends these two toolkits with those features, which are outside the scope of both. The development cycle of an imageguided surgery navigation system is too long to meet current clinical needs.

This work Lu et al (2012) presents an integrated system developed by the integration of two open source software (IGSTK and MITK). The integration shortens the development cycle of the image-guided surgery navigation system and save human resources simultaneously. An image-guided surgery navigation system was established by connecting the two aforementioned open-source software libraries. Seitel et al (2012) used the Medical Imaging Interaction Toolkit (MITK) as a framework providing image processing tools for the image-guided surgery navigation system of medical imaging software with a high degree of interaction and used the Image-Guided Surgery Toolkit (IGSTK) as a library that provided the basic components of the system for location, tracking, and registration. The electromagnetic tracking device was used to measure the real-time position of surgical tools and fiducials attached to the patient's anatomy. IGSTK was integrated into MITK; at the same time, the compatibility and the stability of this system were emphasized. Experiments showed that an integrated system of the image-guided surgery navigation system could be developed in 2 months. 
The integration of IGSTK into MITK is feasible. Several techniques for 3D reconstruction, geometric analysis, mesh generation, and surface data analysis for medical image analysis of MITK can connect with the techniques for location, tracking, and registration of IGSTK. This integration of advanced modalities can decrease software development time and emphasize the precision, safety, and robustness of the image-guided surgery navigation system.

\section{NiftyRec}

NiftyRec is a software project developed at UCL London, which provides code for tomographic reconstruction (Assaf and Alexander, 2014). Lung registration used the package of NiftyReg. The NiftyReg package contains a global as well as a local registration algorithm. The work of Modat et al (2010) describes the implementation that has been used by the Niftyreggers team, as well as the results. The registrations were performed using a block-matching approach and the free-form deformation algorithm for global and local registration respectively. The average total registration time is just under 6 minutes for the GPU-based implementation, so it should be possible to register the 10 datasets on-site in approximately 1 hour, well within the 3-hour time limit.

\section{NiftySim}

NiftySim is a high-performance nonlinear finite element solver, developed at University College London. A key feature is the option of GPU-based execution, which allows the solver to significantly out-perform equivalent commercial packages. NiftySim, an open-source finite element toolkit, has been designed to allow incorporation of high-performance soft tissue simulation capabilities into biomedical applications. The toolkit provides the option of execution on fast graphics processing unit (GPU) hardware, numerous constitutive models and solidelement options, membrane and shell elements, and contact modelling facilities, in a simple to use library.

The toolkit is founded on the total Lagrangian explicit dynamics (TLEDs) algorithm, which has been shown to be efficient and accurate for simulation of soft tissues. The base code is written in $\mathrm{C}++$, and GPU execution is achieved using the NVidia CUDA framework. In most cases, interaction with the underlying solvers can be achieved through a single Simulator class, which may be embedded directly in third-party applications such as, surgical guidance systems. Advanced capabilities such as contact modelling and nonlinear constitutive models are also provided, as are more experimental technologies like reduced order modelling. A consistent description of the underlying solution algorithm, its implementation with a focus on GPU execution, and examples of the toolkit's usage in biomedical applications are provided by (Johnsen et al, 2015). Efficient mapping of the TLED algorithm to parallel hardware results in very high computational performance. Far exceeding is available in commercial packages. In conclusion, the NiftySim toolkit provides high-performance soft tissue simulation capabilities using GPU technology for biomechanical simulation research applications in medical image computing, surgical simulation, and surgical guidance applications.

\section{Camino}

Camino is a software toolkit for diffusion MRI processing, it is able to do construction of processing pipelines that include modules from other software. At present, the microstructure imaging group at UCL lead development is doing the maintenance of the toolkit. Diffusion-MRI is a rapidly evolving research field that has produced a wealth of algorithms for the analysis of white matter fiber architecture and disorders in the brain. Camino is a free, open-source toolkit 
designed to make a selection of this technology available and convenient to use for the diffusion MRI research community.

Camino implements a data processing pipeline, which allows for easy scripting and flexible integration with other software. The research work summarizes the features of Camino at each stage of the pipeline from the raw data to the statistics used by clinicians and researchers. Design Camino is written in Java, and designed for a Unix-style interface. The user documentation (Cook et al, 2006) is in the form of Unix manual pages, and each program has a shell wrapper, so users do not require any knowledge of Java. The data pipeline provides flexibility, by allowing data to be imported and exported to other software, and transparency, because the output of each Camino program can be analyzed in detail. Figure 1 illustrates the pipeline. Camino processes all data in voxel order, where the measurements for each voxel are stored together. This ordering facilitates the data pipeline model and allows each voxel to be processed independently, which simplifies parallel processing. All of Camino's output is in a documented raw binary format. The tractography module optionally outputs Analyze images for easy integration with visualization software.

\section{DTI-TK}

DTI-TK is a spatial normalization and atlas construction toolkit optimized for examining white matter morphometric using DTI data. This software developed by Gary Zhang. According to Keihaninejad et al (2013) a publication has been published in Neuro Image. It ranked DTI-TK as the top-performing tool in its class. In recent years, diffusion tensor imaging (DTI) has become the modality of choice to investigate white matter pathology in the developing brain. To study neonate Crabbe disease with DTI, we evaluate the performance of linear and non-linear DTI registration algorithms for atlas based fiber tract analysis. The DTI scans of 10 age-matched neonates with infantile Crabbe disease are mapped into an atlas for the analysis of major fiber tracts. The genu and splenium of the corpus callosum, the internal capsules tracts and the uncinated fasciculi. The neonate atlas is based on 377 healthy control subjects, generated using an unbiased diffeomorphic atlas building method. To evaluate the performance of one linear and seven nonlinear commonly used registration algorithms for DTI we propose the use of two novel evaluation metrics: a regional matching quality criterion incorporating the local tensor orientation similarity, and a fiber property profile based metric using normative correlation. Our experimental results indicate that the whole tensor based registration method within the DTIToolkit (DTI-TK) shows the best performance for our application.

\section{RESULT AND DISCUSSION}

In this section, the survey of the fifteen medical image processing tools is tabulated in Table I. Analysis is done based on the following criteria. The table 1 shows comparison between the fifteen medical image processing tools listed. 
Table 1. Comparison of Image Processing Tools

\begin{tabular}{|c|c|c|c|c|c|c|c|c|c|c|c|c|c|c|c|c|}
\hline \multicolumn{2}{|c|}{ Image Processing Tool } & 5 & $\underline{E}$ & ॠ & $\sum_{\bar{n}}$ & $\sum_{0}^{\infty}$ & 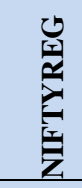 & 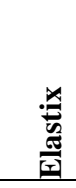 & $\frac{2}{2}$ & 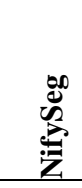 & 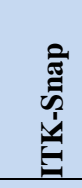 & $\underline{E}$ & $\frac{\mathscr{d}}{3}$ & 荡 & ્ֶ. & 峘 \\
\hline \multicolumn{2}{|c|}{ Latest supported version } & 6.1 & 4.0 & 5.0 & 12 & 1.5 & 3.1 & 4.7 & 2.1 & 3.1 & 3.2 & 03 & 1.6 .9 & 2.0 & 2.0 & 3.0 \\
\hline \multicolumn{2}{|c|}{ Date of last published } & 2014 & 2014 & 2014 & 2014 & 2013 & 2013 & 2014 & 2014 & 2014 & 2014 & 2014 & 2014 & 2014 & 2013 & 2011 \\
\hline \multicolumn{2}{|c|}{ System Interface (GUI) } & $\mathrm{x}$ & $\mathrm{x}$ & $\mathrm{x}$ & $\mathrm{x}$ & $\mathrm{x}$ & $\mathrm{x}$ & $\mathrm{x}$ & $\mathrm{x}$ & $\mathrm{x}$ & $\mathrm{x}$ & $\mathrm{x}$ & $\mathrm{x}$ & $\mathrm{x}$ & $\mathrm{x}$ & $\mathrm{x}$ \\
\hline \multirow{9}{*}{ 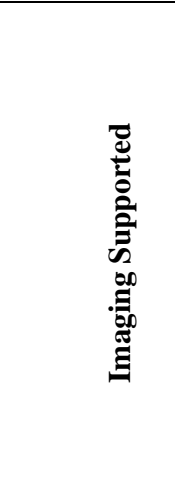 } & MRI & & $\mathrm{x}$ & $\mathrm{x}$ & $\mathrm{x}$ & $\mathrm{x}$ & $\mathrm{x}$ & $\mathrm{x}$ & $x$ & $\mathrm{x}$ & & $\mathrm{x}$ & $\mathrm{x}$ & $\mathrm{x}$ & $\mathrm{x}$ & $\mathrm{x}$ \\
\hline & Ultrasound & & & & & $\mathrm{x}$ & $\mathrm{x}$ & $\mathrm{x}$ & & $\mathrm{x}$ & & $\mathrm{x}$ & & $\mathrm{x}$ & & $\mathrm{x}$ \\
\hline & X-ray & & & & & $\mathrm{x}$ & $\mathrm{x}$ & $\mathrm{x}$ & & $\mathrm{x}$ & & $\mathrm{x}$ & & & & \\
\hline & fMRI & & & $\mathrm{x}$ & $\mathrm{x}$ & $\mathrm{x}$ & & $\mathrm{x}$ & $\mathrm{x}$ & & & $\mathrm{x}$ & & & $\mathrm{x}$ & \\
\hline & PET & & & & $\mathrm{x}$ & $\mathrm{x}$ & & $\mathrm{x}$ & & & & $\mathrm{x}$ & & & & \\
\hline & CT-Scan & & & & $\mathrm{x}$ & $\mathrm{x}$ & & $\mathrm{x}$ & & & & $\mathrm{x}$ & $\mathrm{x}$ & $\mathrm{x}$ & & $\mathrm{x}$ \\
\hline & EEG & & & & $\mathrm{x}$ & $x$ & & $\mathrm{x}$ & & & & $\mathrm{x}$ & & & & \\
\hline & Mammogram & & & & & $\mathrm{x}$ & $\mathrm{x}$ & $\mathrm{x}$ & & $\mathrm{x}$ & & $\mathrm{x}$ & & & $\mathrm{x}$ & \\
\hline & 3D Images & $\mathrm{x}$ & & & & $\mathrm{x}$ & & $\mathrm{x}$ & & $\mathrm{x}$ & $\mathrm{x}$ & $\mathrm{x}$ & $\mathrm{x}$ & & & \\
\hline \multirow{7}{*}{ 氖 } & Generic & $\mathrm{x}$ & $\mathrm{x}$ & $\mathrm{x}$ & $\mathrm{x}$ & $\mathrm{x}$ & & $\mathrm{x}$ & $\mathrm{x}$ & $\mathrm{x}$ & & & & & $\mathrm{x}$ & \\
\hline & Registration & & $\mathrm{x}$ & & $\mathrm{x}$ & $\mathrm{x}$ & $\mathrm{x}$ & $\mathrm{x}$ & $\mathrm{x}$ & $\mathrm{x}$ & & $\mathrm{x}$ & & & $\mathrm{x}$ & \\
\hline & Segmentation & & $\mathrm{x}$ & & $\mathrm{x}$ & $\mathrm{x}$ & & $\mathrm{x}$ & & $\mathrm{x}$ & & $\mathrm{x}$ & $\mathrm{x}$ & $\mathrm{x}$ & $\mathrm{x}$ & $\mathrm{x}$ \\
\hline & Visualisation & $\mathrm{x}$ & & & & $\mathrm{x}$ & $\mathrm{x}$ & $\mathrm{x}$ & & & $\mathrm{x}$ & $\mathrm{x}$ & $\mathrm{x}$ & $\mathrm{x}$ & $\mathrm{x}$ & $\mathrm{x}$ \\
\hline & Reconstruction & $\mathrm{x}$ & & $\mathrm{x}$ & $\mathrm{x}$ & $\mathrm{x}$ & & $\mathrm{x}$ & $\mathrm{x}$ & & $\mathrm{x}$ & & $\mathrm{x}$ & $\mathrm{x}$ & & $\mathrm{x}$ \\
\hline & Simulation & $\mathrm{x}$ & & $\mathrm{x}$ & & $\mathrm{x}$ & & $\mathrm{x}$ & & & $\mathrm{xx}$ & & $\mathrm{x}$ & $\mathrm{x}$ & & $\mathrm{x}$ \\
\hline & Diffusion & $\mathrm{x}$ & & & $\mathrm{x}$ & $\mathrm{x}$ & & $\mathrm{x}$ & & & & $\mathrm{x}$ & $\mathrm{x}$ & & $\mathrm{x}$ & \\
\hline \multirow{3}{*}{ 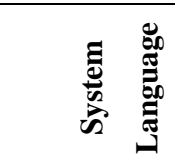 } & $\mathrm{C \#}$ & & & $\mathrm{x}$ & & & & & & & $\mathrm{x}$ & & & & & \\
\hline & $\mathbf{C}$ & & & & $\mathrm{x}$ & & & & & & & $\mathrm{x}$ & $\mathrm{x}$ & & & \\
\hline & $\mathrm{C}++$ & $\mathrm{x}$ & & & & $x$ & & $\mathrm{x}$ & & & & & & & & \\
\hline
\end{tabular}




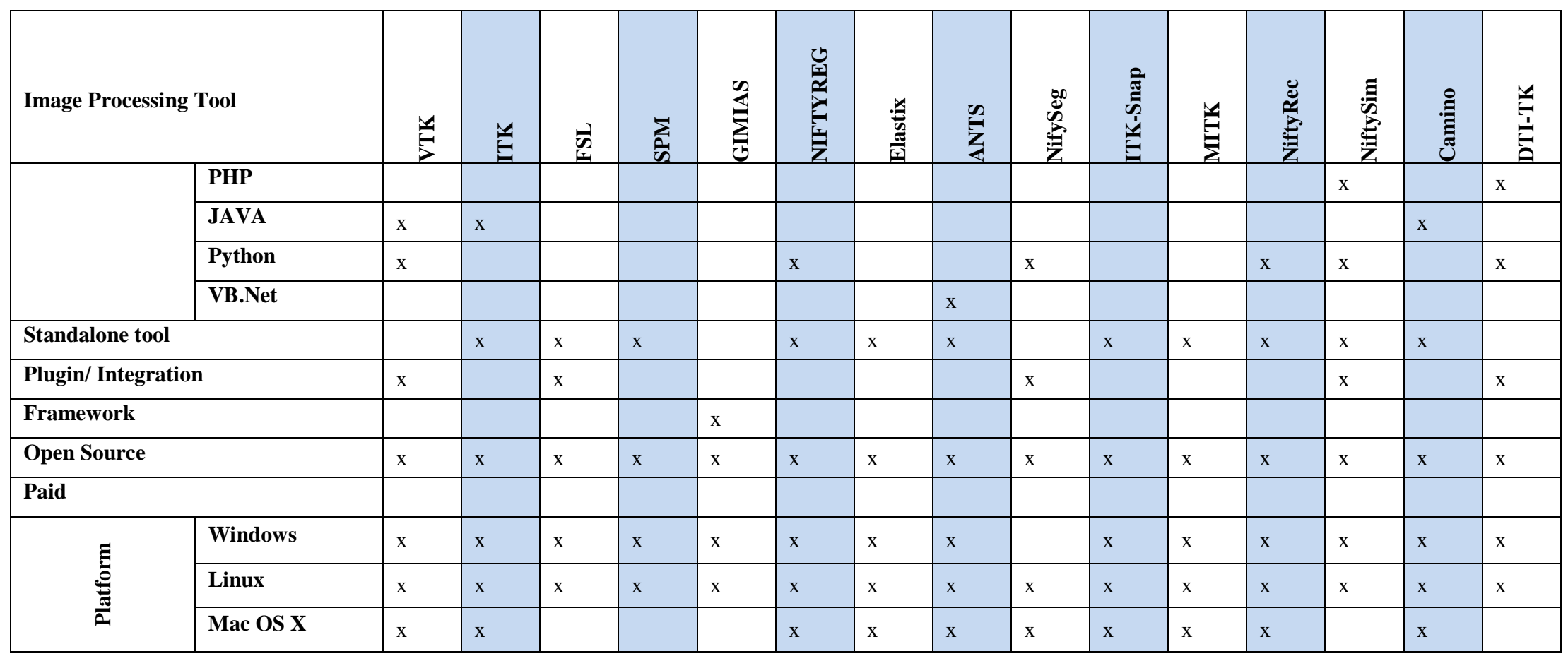


Comparisons between the fifteen medical image processing tools are tabulated as in table 1 . The criteria for the comparisons are the latest versions of tool supported, system GUI supported, medical imaging (modalities) supported, tool function provided, languages of tools, type of tools, price and the type of platform to run in.

The first criterion compared is the latest version of image processing tool supported. In this criterion, VTK, ITK, SPM, GIMIAS, Elastix and MITK stood up for providing the latest software version standard to the users. In terms of tools' GUI, all fifteen type of tools provided interface control for users, thus the existence of GUI show user-friendly and convenient to beginner. All fifteen medical image processing tools have different supporting modalities. For instance, VTK only support 3D medical imaging, where SPM able to support 5 types of medical imaging which is MRI, fMRI, PET, CT-Scan, EEG. .Meanwhile, GIMIAS, Elastix and MITK support all type of medical images.

Moreover, each tool has different functionality, for example, NiftyReg have the least utility, it only support segmentation and visualization, while Elastix and Camino provided all functions in their tool. Followed by next discussion topic, which is the language used to develop the software tool. FSL, ITK-Snap, Camino and DTI-TK are C\# tools, on the other hand, SPM, MITK and NiftySeg are language $\mathrm{C}$ tools. $\mathrm{C}++$ tools are VTK, GIMIAS and Elastix. ITK, Camino and DTI_TK are JAVA tools, the rest tools like NiftyReg, NiftySeg, NiftyRec and NiftySim are python tools. Only ANTS built in VB.Net

All the medical image processing tools surveyed can work as a standalone tool while, VTK, FSL and NifySeg provides plugin or integration with another IDE tool such as Matlab. Only GIMIAS is framework. Most of the MIP tools surveyed have free version to be used noncommercially, while others have free trial versions which expires after one to three months of usage. For some of the MIP tools to be fully utilized, it requires users to purchase a license to unlock the full function of the MIP tool. Most of the MIP tools can be installed in cross platform (Windows, Linux and Mac OS X) as long as that particular platform supports Java.

Based on the survey and user experience, the author would like to recommend Elastix for medical image processing, it is due to the tool provides the most user friendly and complete experience. It allow user to download the latest software tool version and the software have GUI interface. It also supports all type of imaging like MRI, ultrasound, X-ray and CT-scan. It supports a wide variety of function when performing image analysis and this tool can run as a standalone tool or be integrated with a wide variety of IDE such as Matlab, NetBeans, and Visual Studio.

Elastix is an open source tool, user can download the latest version in their official web site, besides that, the web site also providing tutorials for beginner. Elastix is a complete MIP tools, it also has a wide option for importing and exporting result. For importing files, it supports Dicom, JPEG (.jpg), Bitmap (.bmp), PNG (.Png), and TIFF (.tif) version of medical image. Thus, the Elastix medical image processing tool provides a really complete function as an MIP tool with its wide variety of function provided and file type imports, exports and a lot of platform supported.

For users looking for a full featured experience, it is recommended to try out the Elastix for MIP tool. While users who are looking to just try out a segmentation or visualisation tool, it is recommended to try out the Camino. Camino is similar to Elastix, it supports all type of medical imaging and also provided all the listed function; however it only can be installed in window. Although Camino does not provide installer in other operating system, it serves as a good basic functional tool. Most of the MIP tools that are free for non-commercial use are marked as free from the table 1. Free downloads are available from the respective tools' website. For the MIP tools that are not marked as free, it means that it requires user to purchase a license to use the particular MIP tool. 


\section{CONCLUSION}

The following conclusions can be drawn from this research:

i. Elastix and Camino is a potential tool in medical image processing.

ii. Gimias is another medical image processing tool that provided in framework.

iii. In this research, a survey paper has been completed with reviewing of 15 tools

In conclusion, this paper surveys on the existing tools of medical image processing and recommends several medical image processing tools to be applied on medical image analysis. The scope only includes medical image tools. Each medical image processing (MIP) tool has its own limitations. Hence this paper can be used as a reference. However, there are certain limitations for this review paper which is this paper only includes 15 MIP tools. Recommendation for future works includes improving the number of survey tool in the next paper.

\section{ACKNOWLEDGMENTS}

We would like to express our gratitude to the university in providing a platform for our research. Other than that, we are also grateful for Malaysia's Department of Higher Education in providing the Fundamental Research Grant Scheme for our research. Last but not least, we would also like to express our appreciation to all the previous researchers for their publications which helped a lot in our research for this paper. Without going through their publications, we would not have produced this paper.

\section{REFERENCES}

Alexander, G. E., Chen, K., Pietrini, P., Rapoport, S. I., \& Reiman, E. M. (2002). Longitudinal PET evaluation of cerebral metabolic decline in dementia: a potential outcome measure in Alzheimer's disease treatment studies. American Journal of Psychiatry, 159(5), 738745.

Assaf, Y., \& Alexander, D. C. (2014). Advanced Methods to Study White Matter Microstructure.

Awad, T. S., Moharram, H. A., Shaltout, O. E., Asker, D., \& Youssef, M. M. (2012).

Applications of ultrasound in analysis, processing and quality control of food: A review. Food Research

International, 48(2),

410-427.

Avants, B. B., Tustison, N., \& Song, G. (2009). Advanced normalization tools (ANTS). Insight J, 2, 1-35.

Avants, B. B., Tustison, N. J., Song, G., Cook, P. A., Klein, A., \& Gee, J. C. (2011). A reproducible evaluation of ANTs similarity metric performance in brain image registration. Neuroimage, 54(3), 2033-2044.

Berg, W. A., Zhang, Z., Lehrer, D., Jong, R. A., Pisano, E. D., Barr, R. G., ... \& Morton, M. J. (2012). Detection of breast cancer with addition of annual screening ultrasound or a single screening MRI to mammography in women with elevated breast cancer risk. Jama, 307(13), 1394-1404.

Cook, P. A., Bai, Y., Nedjati-Gilani, S. K. K. S., Seunarine, K. K., Hall, M. G., Parker, G. J., \& Alexander, D. C. (2006, May). Camino: open-source diffusion-MRI reconstruction and processing. In 14th scientific meeting of the international society for magnetic resonance in medicine (Vol. 2759). Seattle WA, USA.

Drzezga, A., Souvatzoglou, M., Eiber, M., Beer, A. J., Fürst, S., Martinez-Möller, A., ... \& Schwaiger, M. (2012). First clinical experience with integrated whole-body PET/MR: 
comparison to PET/CT in patients with oncologic diagnoses. Journal of Nuclear Medicine, 53(6), 845-855.

DTI registration in atlas based fiber analysis of infantile Krabbe disease. Neuroimage, 55(4), $1577-1586$.

Fagiolo, G., Waldman, A., \& Hajnal, J. V. (2014). A simple procedure to improve FMRIb software library brain extraction tool performance. The British journal of radiology.

Gharge, S. (2013). Segmentation of medical images.

Greenberg, A. K., Lu, F., Goldberg, J. D., Eylers, E., Tsay, J. C., Yie, T. A., ... \& AddrizzoHarris, D. (2012). CT scan screening for lung cancer: risk factors for nodules and malignancy in a high-risk urban cohort. PloS one, 7(7), e39403.

Hanwell, M. D., Martin, K. M., Chaudhary, A., \& Avila, L. S. (2015). The Visualization Toolkit (VTK): Rewriting the rendering code for modern graphics cards. SoftwareX, 1, 9-12.

Johnsen, S. F., Taylor, Z. A., Clarkson, M. J., Hipwell, J., Modat, M., Eiben, B., ... \& Ourselin, S. (2015). NiftySim: A GPU-based nonlinear finite element package for simulation of soft tissue biomechanics. International journal of computer assisted radiology and surgery, 10(7), 1077-1095.

Johnson, H. J., McCormick, M. M., \& Ibanez, L. (2014). The ITK Software Guide Book 1: Introduction and Development Guidelines Fourth Edition Updated for ITK version 4.7.

Keihaninejad, S., Zhang, H., Ryan, N. S., Malone, I. B., Modat, M., Cardoso, M. J., ... \& Ourselin, S. (2013). An unbiased longitudinal analysis framework for tracking white matter changes using diffusion tensor imaging with application to Alzheimer's disease. NeuroImage, 72, 153-163.

Larrabide, I., Omedas, P., Martelli, Y., Planes, X., Nieber, M., Moya, J. A., ... \& Bijnens, B. H. (2009). GIMIAS: an open source framework for efficient development of research tools and clinical prototypes. In Functional Imaging and Modeling of the Heart (pp. 417-426). Springer Berlin Heidelberg.

Lee, L. K., Liew, S. C., \& Thong, W. J. (2015). A review of image segmentation methodologies in medical image. In Advanced computer and communication engineering technology (pp. 1069-1080). Springer International Publishing.

Liu, Y., Kot, A., Drakopoulos, F., Yao, C., Fedorov, A., Enquobahrie, A., ... \& Chrisochoides, N. P. (2014). An ITK implementation of a physics-based non-rigid registration method for brain deformation in image-guided neurosurgery.

Lu, T., Liang, P., Wu, W. B., Xue, J., Lei, C. L., Li, Y. Y., ... \& Liu, F. Y. (2012). Integration of the image-guided surgery toolkit (igstk) into the medical imaging interaction toolkit (mitk). Journal of digital imaging, 25(6), 729-737.

Kerner, G. S., Fischer, A., Koole, M. J., Pruim, J., \& Groen, H. J. (2015). Evaluation of elastixbased propagated align algorithm for VOI-and voxel-based analysis of longitudinal 18FFDG PET/CT data from patients with non-small cell lung cancer (NSCLC). EJNMMI research, 5(1), 15.

Khesin, M., Quenan, D., Jesikiewicz, T., Kenien, D., \& Girvan, R. (1997). Demonstration tests of new burner diagnostic system on a $650 \mathrm{MW}$ coal-fired utility boiler (No. CONF970456--). Illinois Inst. of Tech., Chicago, IL (United States).

Mengler, L., Khmelinskii, A., Diedenhofen, M., Po, C., Staring, M., Lelieveldt, B. P., \& Hoehn, M. (2014). Brain maturation of the adolescent rat cortex and striatum: changes in volume and myelination. Neuroimage, 84, 35-44.

Modat, M., McClelland, J., \& Ourselin, S. (2010). Lung registration using the NiftyReg package. Medical Image Analysis for the Clinic-A Grand Challenge, 2010, 33-42. 
Nolden, M., Zelzer, S., Seitel, A., Wald, D., Müller, M., Franz, A. M., ... \& Maier-Hein, K. H. (2013). The medical imaging interaction toolkit: challenges and advances. International journal of computer assisted radiology and surgery, 8(4), 607-620.

Penny, W. D., Friston, K. J., Ashburner, J. T., Kiebel, S. J., \& Nichols, T. E. (Eds.). (2011). Statistical parametric mapping: the analysis of functional brain images: the analysis of functional brain images. Academic press.

Pettit, C., Bishop, I., Sposito, V., Aurambout, J. P., \& Sheth, F. (2012). Developing a multi-scale visualisation framework for use in climate change response. Landscape ecology, 27(4), 487-508.

Qasrawi, R., \& Ivorra, A. (2015). Impact of Liver Vasculature on Electric Field Distribution during Electroporation Treatments: An Anatomically Realistic Numerical Study. In 6th European Conference of the International Federation for Medical and Biological Engineering (pp. 573-576). Springer International Publishing.

Seitel, A., Yung, K., Mersmann, S., Kilgus, T., Groch, A., dos Santos, T. R., ... \& Maier-Hein, L. (2012). MITK-ToF-Range data within MITK. International journal of computer assisted radiology and surgery, 7(1), 87-96.

Smith, S. M., Jenkinson, M., Woolrich, M. W., Beckmann, C. F., Behrens, T. E., Johansen-Berg, H., ... \& Niazy, R. K. (2004). Advances in functional and structural MR image analysis and implementation as FSL. Neuroimage 23 (Suppl. 1), S208-S219. External Resources Pubmed/Medline (NLM) CrossRef (DOI).

Solomon, C., \& Breckon, T. (2011). Fundamentals of Digital Image Processing: A practical approach with examples in Matlab. John Wiley \& Sons.

Sonka, M., Hlavac, V., \& Boyle, R. (2014). Image processing, analysis, and machine vision. Cengage Learning.

Sowell, E. R., Levitt, J., Thompson, P. M., Holmes, C. J., Blanton, R. E., Kornsand, D. S., ... \& Toga, A. W. (2000). Brain abnormalities in early-onset schizophrenia spectrum disorder observed with statistical parametric mapping of structural magnetic resonance images. American Journal of Psychiatry, 157(9), 1475-1484.

Stein, E. A., Pankiewicz, J., Harsch, H. H., Cho, J. K., Fuller, S. A., Hoffmann, R. G., ... \& Bloom, A. S. (1998). Nicotine-induced limbic cortical activation in the human brain: a functional MRI study. American Journal of Psychiatry.

Suryanarayana, C., \& Norton, M. G. (2013). X-ray diffraction: a practical approach. Springer Science \& Business Media.

TIG. (2014). The TIG, Image processing tool. Retrieved from http://cmictig.cs.ucl.ac.uk/wiki/index.php/Main_Page.

User-guided 3D active contour segmentation of anatomical structures: significantly improved efficiency and reliability. Neuroimage, 31(3), 1116-1128.

Yushkevich, P. A., Piven, J., Hazlett, H. C., Smith, R. G., Ho, S., Gee, J. C., \& Gerig, G. (2006). 